\title{
Momentum distribution of a trapped Fermi gas with large scattering length
}

\author{
L. Viverit, ${ }^{1,2}$ S. Giorgini, ${ }^{1}$ L. P. Pitaevskii,,${ }^{1,3}$ and S. Stringari ${ }^{1}$ \\ ${ }^{1}$ Dipartimento di Fisica, Università di Trento and BEC-INFM, I-38050 Povo, Italy \\ ${ }^{2}$ Dipartimento di Fisica Università di Milano, via Celoria 16, I-20122 Milan, Italy \\ ${ }^{3}$ Kapitza Institute for Physical Problems, 117334 Moscow, Russia
}

\begin{abstract}
Using a scattering length parametrization of the BCS-BEC crossover as well as the local density approximation for the density profile, we calculate the momentum distribution of a harmonically trapped atomic Fermi gas at zero temperature. Various interaction regimes are considered, including the BCS phase, the unitarity limit and the molecular regime. We show that the relevant parameter which characterizes the crossover is given by the dimensionless combination $N^{1 / 6} a / a_{h o}$, where $N$ is the number of atoms, $a$ is the scattering length and $a_{h o}$ is the oscillator length. The width of the momentum distribution is shown to depend in a crucial way on the value and sign of this parameter. Our predictions can be relevant for experiments on ultracold atomic Fermi gases near a Feshbach resonance.
\end{abstract}

Recent experiments on ultracold atomic Fermi gases near a Feshbach resonance, have pointed out the crucial role played by two-body interactions. Spectacular effects concern the hydrodynamic behaviour exhibited by the expansion after release of the trap [1, 2, 3] and the evidence of molecular formation for positive values of the scattering length [4, 5]. These experiments open important perspectives towards the realization of the superfluid phase in Fermi gases, whose critical temperature has been predicted to be significantly enhanced by resonance effects [6, 7, 8], and towards the study of the BCS-BEC crossover [9, 10, 11, 12] and of many-body effects in the presence of a Feshbach resonance [13, 14, 15].

The purpose of this paper is to point out that an important diagnostics of the state of the system across the resonance is the atomic momentum distribution whose shape, at low temperature, is very sensitive to the size and sign of the scattering length. The momentum distribution can be directly measured in these systems either by suddenly switching off the scattering length and imaging the expanding atomic cloud [3], or by Bragg spectroscopy [16].

In this paper we will consider a Fermi gas of two spin species. The interspecies interaction is characterized by the s-wave scattering length $a$ which will be assumed to be larger than the effective range $r_{0}$ of the interaction. Furthermore we will also assume that the relative momentum $\hbar k$ between the colliding atoms is smaller than $\overline{/} r_{0}$. If the scattering length $a$ is large and positive a weakly bound molecule forms in the vacuum with binding energy $E_{b}=-\hbar^{2} / m a^{2}$ and size $a$ [17]. A peculiar feature of the resonance is that when $k|a| \gg 1$ the scattering amplitude $f$ is unitarity limited and $f \rightarrow i / k$, independent of the value of $a[18]$.

In the many-body problem the above lengths should be compared with the average distance between particles proportional, at $T=0$, to the inverse of the Fermi wavenumber

$$
k_{F}=\left(6 \pi^{2}\right)^{1 / 3} n_{\sigma}^{1 / 3},
$$

where the densities $n_{\sigma}$ of the two species will be taken equal. By assuming that both the average distance between particles and the modulus of the scattering length are larger than the effective range $r_{0}$, the effects of interactions will not depend on the actual value of $r_{0}$, but only on the combination $k_{F} a$.

For small and negative values of $k_{F} a$, a homogeneous gas at zero temperature exhibits a BCS superfluid phase, whose momentum distribution differs very little from the step function $\theta\left(k_{F}-k\right)$ of the non-interacting Fermi gas.

When either the value of the scattering length or of the density increases, correlations become more and more important. Eventually, in the unitarity limit $k_{F}|a| \gg 1$, the configuration of the system is expected to exhibit a universal behavior, independent of the actual value of $a$ 24]. When we move to the side of the Feshbach resonance where the scattering length is large and positive, bound molecules of size $a$ would form in the vacuum. However if $a$ is still much larger than the interparticle spacing the actual state of the many-body system will be very different. Only when $k_{F}$ a becomes smaller than unity will these molecules not be perturbed by the medium and behave just as independent bosons. In this limit the atomic momentum distribution is expected to become $\sim 1 /\left(k^{2} a^{2}+1\right)^{2}$, corresponding to the momentum distribution of atoms inside molecules.

In the following we shall work at $T=0$ and take into account the effect of the harmonic trapping. This is important because the actual values of the densities, and hence of $k_{F}$, realized in experiments depend in a crucial way on the trapping parameters. We use the model developed in [12] to investigate the BCS-BEC crossover at $T=0$, which accounts for all the regimes introduced above (BCS limit, unitarity limit, BEC molecular limit) with a unique tuning parameter fixed, in uniform gases, by the combination $k_{F} a$. This approach consists of a generalization of the gap equation and the number equation of the usual BCS theory to the whole resonance region, and corresponds to using the saddle point approximation for the zero temperature partition function. 
Eliminating as usual the bare interatomic potential in favor of the scattering t-matrix in the vacuum, one obtains the result

$$
\frac{m}{4 \pi \hbar^{2} a}=\frac{1}{V} \sum_{\mathbf{k}}\left(\frac{1}{2 \epsilon_{k}}-\frac{1}{2 E_{k}}\right)
$$

for the gap equation, where $\epsilon_{k}=\hbar^{2} k^{2} / 2 m, E_{k}=\sqrt{\left(\epsilon_{k}-\mu\right)^{2}+\Delta^{2}}, \mu$ is the chemical potential, $\Delta$ is the gap parameter and $V$ is the volume of the system. The number equation, on the other hand, takes the form

$$
n_{\sigma}=\frac{1}{2 V} \sum_{\mathbf{k}}\left(1-\frac{\epsilon_{k}-\mu}{E_{k}}\right) \text {. }
$$

The two equations form a closed set of equations in the unknowns $\Delta$ and $\mu$ and can be solved for a given value of $k_{F} a$. The approach is reliable when $k_{F} a \rightarrow 0^{ \pm}$with $|a| \gg r_{0}$, and approximate in the region $k_{F}|a| \gtrsim 1$.

It is immediate to see that the particle distribution of atoms of either species is given by the expression

$$
n_{k}=\frac{1}{2}\left(1-\frac{\epsilon_{k}-\mu}{E_{k}}\right),
$$

and corresponds, in the usual Bogoliubov theory of Fermi superfluids, to $n_{k}=\left|v_{k}\right|^{2}$ where $v_{k}$ are the Bogoliubov amplitudes. The above expression for $n_{k}$ is only valid for values of $k$ smaller than the inverse of the effective range $r_{0}$. The values of $k$ can however be much larger than $1 / a$ since we assume $a \gg r_{0}$.

Solutions of equations (2) and (3) are available for the whole range of values of $k_{F} a$ 21, 22. Particularly simple solutions exist in the limits $k_{F} a \rightarrow 0^{ \pm}$. In the BCS limit $\left(k_{F} a \rightarrow 0^{-}\right)$the solutions are well known: $\mu \simeq \epsilon_{F}$ and $\Delta \simeq 8 e^{-2} \epsilon_{F} \exp \left(\pi / 2 k_{F} a\right)$, with $\epsilon_{F}=\hbar^{2} k_{F}^{2} / 2 m$ being the Fermi energy. Notice that, since the present theory neglects the fluctuations in the number-density and spin-density, the Gorkov correction to the gap $\Delta$ is not included [19, 20]. However, this has no major relevance for the calculation of the particle distribution which in this limit is close to the free-gas value.

In the BEC limit instead one finds $\mu \simeq-\hbar^{2} / 2 m a^{2}+2 \pi \hbar^{2} a n_{\sigma} / m$ and $\Delta \simeq(16 / 3 \pi)^{1 / 2} \epsilon_{F} / \sqrt{k_{F} a}$. The first term in the chemical potential is simply $E_{b} / 2$, i.e. the molecular binding energy per atom. By rewriting the second term as $2 \pi \hbar^{2} a_{B} n_{\sigma} / m_{B}$, where $a_{B}=2 a$ and $m_{B}=2 m$, one can see that this accounts for the mean field molecule-molecule interaction energy [28]. The quantity $\Delta$ instead plays the role of an order parameter for the molecular Bose-Einstein condensate (see for example [23]).

In both the BCS and BEC limits $\Delta$ is much smaller than the abolute value of the chemical potential, so that the particle distribution can be expanded in terms of $\Delta /|\mu|$. In the BCS limit the lowest order in the expansion is simply the free Fermi step function, and it is enough to stop at this level for the considerations which follow. In the BEC limit one instead finds the result

$$
n_{\mathbf{k}}=\frac{4}{3 \pi}\left(k_{F} a\right)^{3} \frac{1}{\left(k^{2} a^{2}+1\right)^{2}} .
$$

This is proportional to the square of the Fourier transform of the molecular wavefunction $\psi(r)=(1 / r) \exp (-r / a)$, indicating that in this limit the particle distribution arises from the motion of atoms inside the molecules, and the prefactor accounts for the fact that there are $n_{\sigma}$ molecules per unit volume.

Let us now consider the most interesting unitarity limit where $k_{F} a \rightarrow \pm \infty$. The left hand side of Eq. (2) vanishes, and this reduces to an implicit equation for the ratio $\Delta / \mu$. A straightforward calculation [21, 22] gives $\Delta / \mu \simeq 1.16$ and from Eq. (3) one finally finds the results $\Delta \simeq 0.69 \epsilon_{F}$ and $\mu \simeq 0.59 \epsilon_{F}$. In the unitarity limit the proportionality of the chemical potential and of $\Delta$ with $\epsilon_{F}$ can be deduced from general dimensional arguments and follows from the fact that $a$ is no longer a relevant scale in the problem. In this limit the chemical potential is often written in the form $\mu=(1+\beta) \epsilon_{F}$ where, in our case, $\beta \simeq-0.41$. It is interesting to compare this prediction with the results of more elaborated approaches. A recent microscopic calculation based on quantum Monte-Carlo techniques gives the value $\beta=-0.56$ [25], while the theory of resonance superfluidity based on saddle-point approximation yields the value $\beta \simeq-0.35[26$. The value of $\beta$ has also been the object of recent experimental investigations in trapped Fermi gases at finite temperature, through the study of the release energy [1, 3].

The predictions of the theory for the particle distribution of a uniform gas are reported in Fig. 1] which shows that during the transition from the ideal gas (dashed line) to the molecular limit (full line) across the unitarity limit (long-dashed line) the atomic momentum distribution varies in a dramatic way, reflecting the critical role played by two-body interactions. This behavior of the momentum distribution was first pointed out in [10, 11]. More recently, it has been investigated in [27] and, in the unitarity limit, by the authors of [25]. In particular the results of [25] rather 
well agree with the curve of Fig. 1 for the unitarity limit. The width of the momentum distribution ranges from $\sim k_{F}$ in the BCS limit to $\sim 1 / a$ in the BEC regime. Notice that for the value $k_{F} a=0.5$ reported in the figure, the momentum distribution calculated by solving Eqs. (2) and (33), is practically indistinguishible from the "molecular" formula (5).

In trapped configurations we shall calculate the momentum distribution by introducing the local semiclassical particle distribution

$$
n_{\mathbf{k}}(\mathbf{r})=\frac{1}{2}\left(1-\frac{\epsilon_{k}-\mu(\mathbf{r})}{E_{k}(\mathbf{r})}\right),
$$

where $\Delta(\mathbf{r})$ and $\mu(\mathbf{r})$ are obtained by solving Eq. (2) and (3) with the local value of the density. The momentum distribution is then evaluated by integrating the particle distribution in coordinate space:

$$
n(\mathbf{k})=\int \frac{d^{3} r}{(2 \pi)^{3}} n_{\mathbf{k}}(\mathbf{r})
$$

The momentum distribution coincides with the particle distribution function only in a uniform system, where one has the simple relation $n(\mathbf{k})=V n_{\mathbf{k}}$. A fundamental ingredient for the calculation is the density distribution, whose shape can vary very much depending on the regime considered and on the trapping parameters [29]. In the following the density profile will be determined using a local density approximation, based on the solution of the equation

$$
\mu=\mu\left(n_{\sigma}(\mathbf{r})\right)+V_{e x t}(\mathbf{r}) .
$$

In the deep BCS limit the particle distribution is given by the free distribution. For harmonic trapping one finds the well known results $n_{\sigma}(\mathbf{r})=n_{\sigma}(0)\left[1-\left(r / R_{T F}^{0}\right)^{2}\right]^{3 / 2}$ for the density profile, where $R_{T F}^{0}=\left(48 N_{\sigma}\right)^{1 / 6} a_{h o}$ is the Thomas-Fermi radius, and $n_{\sigma}(0)=\left(4 N_{\sigma} / 3 \pi^{4}\right)^{1 / 2} a_{h o}^{-3}$ is the central density. $N_{\sigma}$ is the number of fermions in each internal state, and $a_{h o}=\sqrt{\hbar / m \omega}$ the harmonic oscillator length, with $\omega=\left(\omega_{x} \omega_{y} \omega_{z}\right)^{1 / 3}$. The momentum distribution takes the following form

$$
n(\mathbf{k})=\frac{\left(R_{T F}^{0}\right)^{3}}{6 \pi^{2}}\left(1-\left(\frac{k}{k_{F}^{0}}\right)^{2}\right)^{3 / 2},
$$

where the Fermi wavenumber satisfies the relation

$$
k_{F}^{0} a=(48)^{1 / 6} N_{\sigma}^{1 / 6} \frac{a}{a_{h o}},
$$

and coincides with the value obtained using Eq. (11) with the central density $n_{\sigma}(0)$.

In the unitarity limit the density profile has the same form as in the ideal case, since the chemical potential in the uniform system has the same power law dependence on the density $\left(\mu \propto n^{2 / 3}\right)$. The only difference with respect to the ideal case is an overall rescaling factor: $\mu=(1+\beta) \epsilon_{F}$, resulting in a contraction of the Thomas-Fermi radius according to the law $R_{T F}^{0} \rightarrow R_{T F}^{0}(1+\beta)^{1 / 4}$. Similarly, the central density is rescaled according to $n(0) \rightarrow n(0)(1+\beta)^{-3 / 4}$ and consequently the Fermi wavenumber, calculated in the center of the trap, is fixed by

$$
k_{F} a=\frac{(48)^{1 / 6}}{(1+\beta)^{1 / 4}} N_{\sigma}^{1 / 6} \frac{a}{a_{h o}} .
$$

The momentum distribution (7) can then be calculated by integrating (6), employing the corresponding values for $\Delta$ and $\mu$ and the rescaled density profile. After introducing the dimensionless variable $\tilde{r}=r /(1+\beta)^{1 / 4} R_{T F}^{0}$ one finds the result:

$$
\begin{aligned}
n(\mathbf{k}) & =\frac{\left(R_{T F}^{0}\right)^{3}(1+\beta)^{3 / 4}}{4 \pi^{2}} \int_{0}^{1} d \tilde{r} \tilde{r}^{2} \\
& \times\left(1-\frac{\left(k / k_{F}^{0}\right)^{2}-(1+\beta)^{1 / 2} f(\tilde{r})}{\sqrt{\left[\left(k / k_{F}^{0}\right)^{2}-(1+\beta)^{1 / 2} f(\tilde{r})\right]^{2}+(1+\beta)^{-1}\left[f(\tilde{r})\left(\Delta / \epsilon_{F}\right)\right]^{2}}}\right),
\end{aligned}
$$

where $f(\tilde{r})=1-\tilde{r}^{2}, \beta=-0.41$ and $\Delta / \epsilon_{F}=0.69$. 
Let us finally discuss the molecular BEC limit. To the extent that the condition $k_{F} a \ll 1$ is satisfied in the center of the trap, one can use the molecular distribution function (5) everywhere and the resulting momentum distribution will be consequently given by

$$
n(\mathbf{k})=\frac{a^{3} N_{\sigma}}{\pi^{2}} \frac{1}{\left(k^{2} a^{2}+1\right)^{2}} .
$$

Also in this case it is important to calculate the quantity $k_{F} a$ in terms of the relevant parameters of the trap, and to this purpose one has to determine the density profile. The leading density dependent term $2 \pi \hbar^{2} a n_{\sigma} / m$ in the chemical potential arises from the molecule-molecule mean-field interaction. This corresponds to the usual interaction term in the Gross-Pitaevskii theory for bosons interacting with scattering length $a_{B}=2 a$. In this limit the local density approximation yields the $T=0$ Thomas-Fermi profile

$$
n_{\sigma}(\mathbf{r})=n_{\sigma}(0)\left(1-\frac{r^{2}}{R_{T F}^{2}}\right),
$$

where

$$
n_{\sigma}(0)=\frac{1}{4 \pi a a_{h o}^{2}}\left(\frac{15 N_{\sigma} a}{2 a_{h o}}\right)^{2 / 5},
$$

is the central density and $R_{T F}=a_{h o}\left(15 N_{\sigma} a / 2 a_{h o}\right)^{1 / 5}$ is the Thomas-Fermi radius. The use of the local density approximation is justified since the Thomas-Fermi parameter $N_{\sigma} a / a_{h o}$ is much larger than unity. In deriving the above expressions we have assumed that atoms and molecules are trapped with the same oscillator frequency $\omega$. Consequently the boson harmonic oscillator length is given by $a_{h o} / \sqrt{2}$. By evaluating the Fermi momentum at the center of the trap one finally finds

$$
k_{F} a=2.2\left(N_{\sigma}^{1 / 6} \frac{a}{a_{h o}}\right)^{4 / 5} .
$$

The quantity $k_{F} a$ should be sufficiently small in order to apply result (12) for the momentum distribution. It is remarkable that in all of the regimes the product $k_{F} a$, related to the gas parameter $n a^{3}$, is fixed by the combination $N_{\sigma}^{1 / 6} a / a_{h o}$. This quantity then permits to characterize the various regimes exhibited by the interacting Fermi gas. The universality of this combination is a consequence of harmonic trapping, and of the fact that the equation of state $\mu / \epsilon_{F}$, evaluated in the uniform phase, has been assumed to be a function of $k_{F} a$. The above discussion also shows that the value of $k_{F} a$ is rather insensitive to the value of $N_{\sigma}$.

In Fig. 2 we plot the momentum distribution calculated for three different values of $N_{\sigma}^{1 / 6} a / a_{h o}$. The distributions are normalized to unity and momenta are expressed in terms of $k / k_{F}^{0}$ where $k_{F}^{0}$ is the Fermi wavevector of a noninteracting gas. Although these results are based on the approximate theory of [9, 10, 11, 12], they provide a first useful estimate of the momentum distribution which might stimulate new experimental investigations as well as more sophisticated and self-consistent theoretical calculations.

Let us finally discuss the experimental possibilities to measure the momentum distribution and to probe the behavior of the system across the resonance. The technique developed in $[5]$ to quickly turn off the scattering length and to immediately release the trap is well suited for this purpose. If the magnetic field is switched off in times shorter than the inverse of the binding energy $\hbar^{2} / m a^{2}$, the molecules are suddenly dissociated and the atoms will expand ballistically. If instead the scattering length is switched off adiabatically the system will form deeper molecular states characterized by microscopic sizes. The imaging of the expanding atomic cloud provides a direct measurement of the momentum distribution of the initial correlated configuration. An important feature emerging from our results (see Figs. 1 and 2) is the presence of large $k$ components in the momentum distribution, with the consequent suppression of $n(k)$ at small $k$. These large $k$ components, whose presence is more and more pronounced as one leaves the ideal Fermi gas condition $k_{F} a \rightarrow 0^{-}$, affect sizably the release energy of the atomic cloud fixed by the kinetic energy 30 .

If one instead releases the trap by keeping the scattering length on, the scenario of the expansion will be completely different. In this case one expects that the expansion will be governed by the laws of hydrodynamics. This will be the case both at zero temperature, where the system is superfluid, and above $T_{c}$ if $k_{F}|a| \gg 1$ due to the resonant effect in the collisional cross section. Under these conditions the expansion will be anisotropic [31] if the confining trap is not symmetric and the release energy, as well as the shape of the expanding cloud, will be determined by the equation of state of the gas. In the unitarity limit the release energy is given by $E_{R} / N_{\sigma}=(3 / 8)(1+\beta)^{1 / 2}\left(6 N_{\sigma}\right)^{1 / 3} \hbar \omega$, while in the molecular regime the expansion will be similar to the one of a dilute Bose gas and one predicts $E_{R} / N_{\sigma}=$ $(1 / 7)\left(15 N_{\sigma} a / a_{h o}\right)^{2 / 5} \hbar \omega$. 
So far the discussion has been restricted to zero temperature. An interesting question concerns the behavior of the momentum distribution at finite temperatures. For temperatures just above $T_{c}$, the momentum distribution can be determined through the calculation of the single particle Green's function by summing the particle-particle ladder diagrams along the lines of [10]. In particular, for negative scattering length and $k_{F}|a| \ll 1$, where $k_{B} T_{c} \ll \epsilon_{F}$, one finds that the momentum distribution of the free Fermi gas is slightly broadened due to thermal effects. In the opposite molecular regime $\left(a>0\right.$ and $\left.k_{F} a \ll 1\right)$ one recovers the momentum distribution (515) of atoms inside molecules, provided the binding energy of the molecule is much larger than the temperature $\hbar^{2} / m a^{2} \gg k_{B} T$. In fact at the critical temperature for the Bose-Einstein condensation of molecules one finds $k_{B} T /\left|E_{B}\right|=0.22\left(k_{F} a\right)^{2}$, and the thermal dissociation of molecules is negligible if $k_{F} a$ is sufficiently small.

In conclusion, in this paper we have reported a first calculation of the momentum distribution of a harmonically trapped two-component Fermi gas as a function of the scattering length in the BCS-BEC crossover. We expect that the large deviations from the ideal Fermi gas distribution can be addressed experimentally in systems close to a Feshbach resonance.

We thank A. Perali for useful comments.

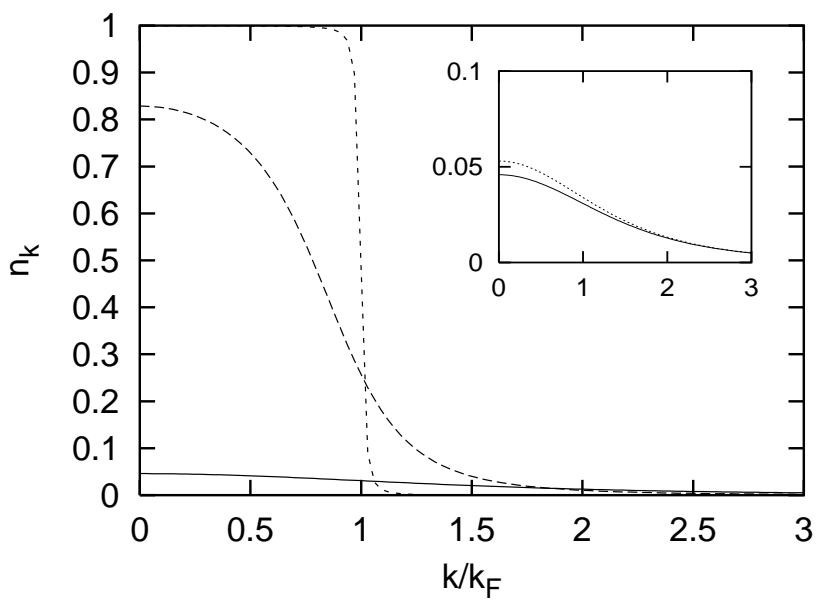

FIG. 1: Particle distribution of a uniform gas in the BEC (solid line), unitarity (long dashed line) and BCS regime (short dashed line). For the BCS regime we have chosen $k_{F} a=-0.5$, and for the BEC one $k_{F} a=0.5$. In the inset the momentum distribution in the BEC regime is compared with the molecular distribution (5) (dotted line). 


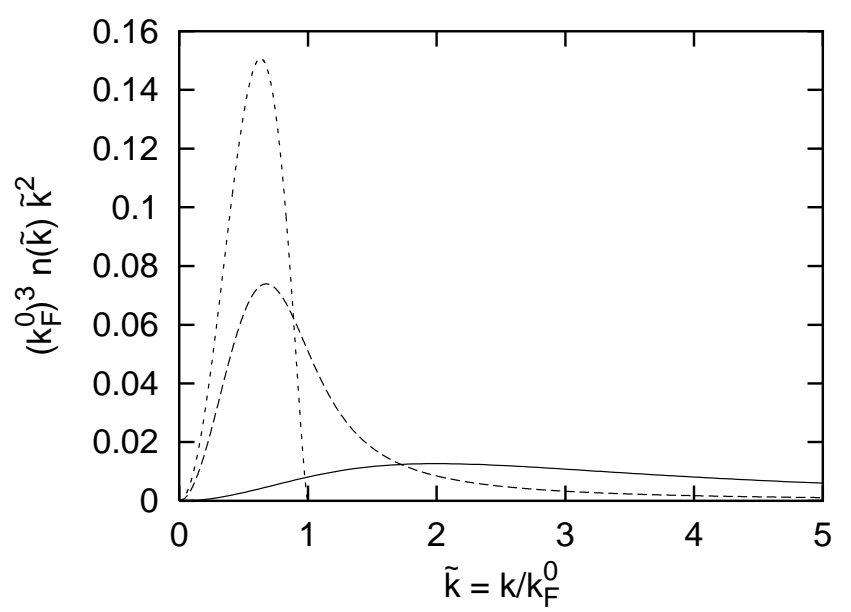

FIG. 2: Momentum distribution of a trapped gas in the BEC (solid line), unitarity (long dashed line) and BCS regime (short dashed line). In the BEC regime we have chosen $N_{\sigma}^{1 / 6} a / a_{h o}=0.26$ corresponding to $k_{F} a=0.75$ (and $k_{F}^{0} a=0.5$ ), while for the BCS regime we have plotted the free fermion distribution. The momentum distributions are multiplied by $k^{2}$ to emphasize the large $k$ behavior and are normalized so that $\int d^{3} k n(k)=1$.

[1] K. M. O'Hara, S. L. Hemmer, M. E. Gehm, S. R. Granade, and J. E. Thomas, Science 298, 2179 (2002).

[2] C. A. Regal and D. S. Jin, Phys. Rev. Lett. 90, 230404 (2003).

[3] T. Bourdel, J. Cubizolles, L. Khaykovich, K. M. F. Magalhaes, S. J. J. M. F. Kokkelmans, G. V. Shlyapnikov, and C. Salomon, cond-mat/0303079

[4] C. A. Regal, C. Ticknor, J. L. Bohn, D. S. Jin, cond-mat/0305028

[5] C. Salomon, private communication.

[6] M. Holland, S. J. J. M. F. Kokkelmans, M. L. Chiofalo, and R. Walser, Phys. Rev. Lett. 87, 120406 (2001).

[7] E. Timmermans, K. Furuya, P. W. Milonni, and A. K. Kerman, Phys. Lett. A 285, 228 (2001).

[8] Y. Ohashi and A. Griffin, Phys. Rev. Lett. 89, 130402 (2002).

[9] A. J. Leggett, in Modern Trends in the Theory of Condensed Matter, edited by A. Pekalski and R. Przystawa, (SpringerVerlag, Berlin, 1980).

[10] P. Nozières and Schmitt-Rink, J. Low Temp. Phys. 59, 195 (1985).

[11] C. A. R. Sá de Melo, M. Randeria and J. R. Engelbrecht, Phys. Rev. Lett. 71, 3202 (1993); M. Randeria, in Bose-Einstein Condensation, edited by Griffin, D. Snoke, and S. Stringari (Cambridge University press, Cambridge, England, 1995).

[12] J. R. Engelbrecht, M. Randeria and C. A. R. Sá de Melo, Phys. Rev. B 55, 15153 (1997).

[13] R. Combescot, cond-mat/0302209

[14] G. M. Bruun and C. J. Pethick, cond-mat/0304535

[15] R. A. Duine and H. T. C. Stoof, cond-mat/0302304

[16] J. Stenger, S. Inouye, A. P. Chikkatur, D. M. Stanper-Kurn, D. E. Pritchard and W. Ketterle, Phys. Rev. Lett. bf 82, 4569 (1999).

[17] Recent experiments on ${ }^{85} \mathrm{Rb}$ and ${ }^{40} \mathrm{~K}$ show that the binding energy of the molecular configurations is only semiquantitatively provided by the law $-\hbar^{2} / m a^{2}$, unless one works close to the resonance, and hence that a more complete description of the two-body problem is in general required.

[18] L. D. Landau and E. M. Lifshitz Quantum mechanics, 3rd edn. (Pergamon, Oxford, 1987).

[19] L. P. Gorkov and T. K. Melik-Barkhudarov, Sov. Phys. JETP 13, 1018 (1961).

[20] H. Heiselberg, C. J. Pethick, H. Smith and L. Viverit, Phys. Rev. Lett. 85, 2418 (2000).

[21] M. Marini, F. Pistolesi and G. C. Strinati, Eur. Phys. J. B 1, 151 (1998).

[22] T. Papenbrock and G. F. Bertsch, Phys. Rev. C 59, 2052 (1999).

[23] P. Pieri and G. C. Strinati, cond-mat/0301023

[24] H. Heiselberg, Phys. Rev. A 63043606 (2001).

[25] J. Carlson, S.Y. Chang, V.R. Pandharipande and K.E. Schmidt, physics/0303094

[26] S.J.J.M.F. Kokkelmans, J.N. Milstein, M.L. Chiofalo, R. Walser and M.J. Holland, Phys. Rev. A 65, 053617 (2002).

[27] Y. Ohashi and A. Griffin, cond-mat/0302196

[28] More sofisticated theories give different predictions for $a_{B}$ (P. Pieri and G. C. Strinati, Phys. Rev. B 61, 15370 (2000); D. S. Petrov and G. V. Shlyapnikov, unpublished), however the results of this paper are not crucially dependent on the 
actual value of $a_{B}$ provided the ratio $a_{B} / a$ is of the order of unity.

[29] A calculation of the evolution of the density profile for the whole range of values of $k_{F} a$ has been recently carried out by A. Perali, P. Pieri, and G. C. Strinati, cond-mat/0212067

[30] The value of the kinetic energy of the atomic cloud cannot be calculated within the present approach since $n(k)$ decreases like $1 / k^{4}$ for large $k$ and consequently the integral $\int d^{3} k k^{2} n(k)$ diverges. This unphysical divergence can be understood recalling that Eq. (4) is only correct for $k \ll 1 / r_{0}$.

[31] C. Menotti, P. Pedri, and S. Stringari Phys. Rev. Lett. 89, 250402 (2002). 